\title{
The Effect of Loss of Speech Audibility on a Measure of Cognitive Function
}

DOI: $10.3766 /$ jaaa.30.10.1

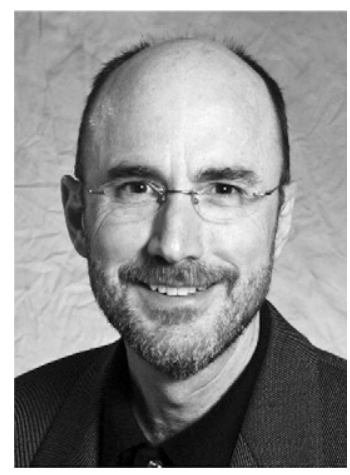

$\mathrm{I}$

t has been estimated that, in the United States, approximately 75 percent of adults over the age of 70 years have hearing impairment ranging from mild to profound (Goman and Lin, 2016). As the population of the world ages, we will have to contend with a larger number of patients who experience ear-related disorders of aging (e.g., presbycusis and presbystasis) and an assortment of diseases that affect older individuals. Dementia is one of those diseases/disorders.

The featured article in this issue of the journal is titled "Effect of Reduced Audibility on Mini-Mental State Examination Scores." For those of you who are unfamiliar with this ubiquitous screening measure, the MiniMental State Examination (MMSE) has 11 sections and can be completed by most individuals in five to 10 minutes. The various functions that are surveyed by the MMSE include time and place orientation, confrontation naming, ability to follow a three-step command, repetition of a phrase, the ability to copy and draw intersecting polygons, and short-term memory. The exam provides up to a total of 30 points and, the higher the score (i.e., usually $>23-26$ points), the less likely the patient has a cognitive impairment.

For years, controversy has centered on issues that are common to the use of screening measures. The main criticism has centered on the trade-off of sensitivity versus efficiency. It has been argued that the MMSE shows ceiling effects and, because of this, it is possible that a patient with mild cognitive impairment might "pass" the screening (i.e., a false negative). One area of research in the use of screening measures has been the effect that sensory impairments, and specifically auditory impairments, have on patient performance.

The paper by Gaeta and colleagues in this issue of the journal represents a clever examination of this issue.
The investigators employed a gender-matched, casecontrol design. There were two groups of subjects. The experimental group was 30 older adults (age range 60-80 years) and the control group consisted of 30 young adults (18-35 years of age). Both groups had to demonstrate normal performance on the Montreal Cognitive Assessment (MoCA). Both the older experimental subjects and the younger control subjects were administered a recorded version of the MMSE that was delivered at a $+25 \mathrm{~dB} \mathrm{~S} / \mathrm{N}$ ratio. Further, control subjects were matched to the experimental subjects and the MMSE was filtered for the control subjects in a manner that approximated the audibility loss of the older subject with whom they were paired. The big question was centered on whether the investigators would observe group differences.

I will let you discover the results of this interesting investigation (they may be different than you might have predicted). I will tell you that the results have made me re-think what information I need to acquire before scoring the MMSE and making (or not making) referrals for further assessments.

The editors hope you enjoy this article and the others that form the last issue in Volume 30 of the Journal of the American Academy of Audiology.

\section{Gary P. Jacobson, PhD} Editor-in-Chief

\section{REFERENCE}

Goman AM, Lin FR. (2016) Prevalence of hearing loss by severity in the United States. Am J Public Health 106:1820-1822. 